\title{
VEHÍCULOS DE GUIADO AUTÓNOMO (AGV) EN APLICACIONES INDUSTRIALES: UNA REVISIÓN
}

\author{
Sergio Alejandro Madrigal Moreno', Nelson David Muñoz Ceballos²
}

${ }^{1}$ Ing. de Control, especialista en Automática. Estudiante de maestría en ingeniería énfasis en gestión de la automatización Politécnico Colombiano Jaime Isaza Cadavid. E-mail: sergio_madrigal54181@elpoli.edu.co ${ }^{2}$ M.Sc. en Automatización y control Industrial. Docente Politécnico Colombiano Jaime Isaza Cadavid. Estudiante de doctorado en ingeniería sistemas e informática, Universidad Nacional de Colombia. E-mail: ndmunoz@elpoli.edu.co

\section{RESUMEN}

Se presenta una revisión del estado del arte de vehículos de guiado autónomo (AGV por sus siglas en inglés: Automated Guided Vehicle) para el transporte de objetos y materia prima en aplicaciones industriales. La revisión de literatura hace énfasis en varios aspectos como la identificación del lugar, aplicación en diferentes entornos, control del vehículo y descripción de los sistemas de software usados para la programación de los robots móviles, de acuerdo a los requisitos o necesidades a resolver. Adicionalmente, se hace un análisis, con base a las referencias citadas, del aporte que hace este tipo de tecnologías no solo a la industria, sino también a otros tipos de modelo de negocio, como lo son el área de la salud, los sistemas de transporte urbano, entre otros.

Palabras clave: Vehículo de guiado autónomo, ROS, Robot, Robot móvil, AGV

Recibido: 31 de Enero de 2019. Aceptado: 25 de Junio de 2019

Received: January 31, 2019 Accepted: June 25, 2019

\section{AUTONOMOUS GUIDED VEHICLES (AGV) IN INDUSTRIAL APPLICATIONS: A REVIEW}

\begin{abstract}
A review of the state of the art is presented of the autonomous guidance vehicles (AGV) to transport objects and raw material in industrial applications. The literature review is focus in different topics as the identification of place, application in different environments, vehicle control and description of the software systems used for the programming of mobile robots, according to the requirements or needs to be resolved. Additionally, an analysis is made, based on the aforementioned references, of the contribution made by this type of technologies not only to the industry, but also to other types of business model, such as the health area, the systems of urban transport, etc.
\end{abstract}

Keywords: Automated Guided Vehicle, ROS, Robot, Mobile robot, AGV.

Cómo citar este artículo: S. Madrigal y N. Muñoz. "Vehículos de guiado autónomo (AGV) en aplicaciones industriales: una revisión", Revista Politécnica, vol. 15, no.28 pp.117-137, 2019. DOI: https://doi.org/10.33571/rpolitec.v15n28a11 


\section{INTRODUCCIÓN}

En los últimos años numerosas industrias manufactureras se han estado modernizando y esto se ha reflejado no solo en la adquisición tecnológica, sino también en la capacidad de llevar todo el volumen de información generado en los procesos hacia los sistemas de gestión, permitiendo consultar el estado de la planta en todo momento, y consecuentemente, habilitando toda la maquinaria para el intercambio de información entre ellas, lográndose así la migración de todo un proceso hacia lo que hoy en día se conoce como la Industria 4.0 (Samaranayake 2017) [1], que no es nada más que la cuarta etapa de las tres revoluciones industriales predecesoras, como se muestra en la figura 1. En síntesis, la Industria 4.0 es una visión de los servicios interconectados para facilitar la innovación y la capacidad de visualizar datos de fabricación, a fin de descubrir nuevas perspectivas para una mayor ventaja competitiva en el sector manufacturero (Hill 2017) [2]. Sin embargo, para llegar a dicho nivel en relación al transporte de mercancía de un sitio a otro y compartiendo información en tiempo real del estado del producto, es necesario estar preparado tecnológicamente, teniendo en cuenta que el mismo nivel de automatización no es asimilado en la logística, donde típicamente los trabajos desempeñados en esta área son realizados por operadores humanos y equipos de accionamiento manual (Vasiljević 2016) [3], sin contar los momentos en que se debe transportar la mercancía de un lugar a otro ya sea por la necesidad de almacenarlos, o porque deben recorrer todo el proceso para ser empacados y despachados al usuario final (Chen 2017) [4].

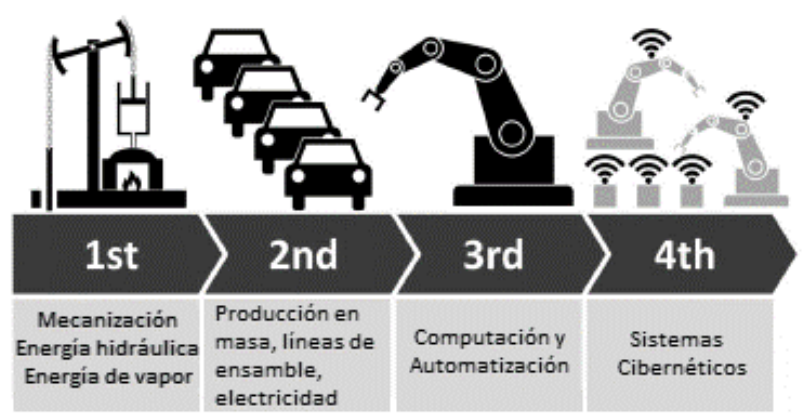

Figura 1. Etapas de desarrollo de la revolución industrial (Hill 2017) [2]
Adicionalmente hay que tener en cuenta la infraestructura del local y distribución interna de la maquinaria ya que esto tiene gran impacto al momento de instalar, ejecutar $\mathrm{y} / \mathrm{o}$ modernizar cualquier tipo de planta industrial (Wang 2015) [5], y más para aquellos procesos que están enfocados en mover o transportar productos de un sitio a otro dentro de su flujo productivo. En ese orden de ideas, existen diferentes tipos de soluciones que permiten trasladar materia prima, productos, mercancía, etc., de un lugar a otro de forma manual o automática. Particularmente, los vehículos de guiado autónomo (AGV) cumplen con esta función, en donde tienen un campo diversificado de aplicaciones que se expande con el tiempo. Los sectores comerciales de aplicación incluyen terminales de contenedores, sistemas de fabricación flexible, almacenes logísticos, agricultura, operaciones militares, gestión de la salud, entre otras (Bechtsis 2017) [6], (Bostelman 2016) [7].

De acuerdo a lo anterior, al momento de diseñar un AGV se debe tener en cuenta las características claves que debe cumplir el vehículo de acuerdo al entorno donde se desempeñará (Le-Anh 2006) [8], por ejemplo, si se quiere transportar material o mercancía pesada de forma automática y sin dependencia humana en un centro logístico, se podria elegir una monta-carga autónoma el cual se ajusta perfectamente para realizar dicha tarea (Andreasson 2015) [9]. En tal sentido, es relevante también seleccionar una adecuada técnica de control para la navegación. Por ejemplo, la lógica difusa es una de ellas, debido a que las reglas heurísticas se actualizan fácilmente y abordar nuevas características que mejoran el desempeño del sistema y por otro lado, el PID (Por sus siglas en inglés: Proportional-Integral-Derivative) también se utiliza en muchos sistemas de control automático, ya sea con parámetros estáticos o dinámicos (Shakya 2014) [10] como en los actuadores de un AGV. Del mismo modo, un método combinado de ambos es adecuado para aplicaciones industriales (Zulfatman 2009) [11] o en un $A G V$ que conserve una velocidad constante independiente de la variación del peso de producto a trasladar (Riesa 2017) [12].

En esta revisión de literatura se estudian los vehículos de guiado autónomo para la solución del transporte de mercancía $\mathrm{y} / \mathrm{o}$ productos en diferentes sectores, haciendo especial énfasis en el sector industrial, y su relación con la industria 4.0. 
El documento se organiza de la siguiente manera: luego de la introducción, en la segunda sección se explica la terminología básica usada en el diseño de un AGV. En la tercera sección se presentan aspectos del estado del arte en donde se explicará la aplicabilidad de esta tecnología desde la identificación del lugar de implantación, desempeño en diferentes entornos de trabajo, algunas técnicas de control y navegación usadas en los AGV y se hace énfasis en el software ROS (por sus siglas en inglés: Robot Operating System). Posteriormente en la cuarta sección se hace un análisis del material revisado. Finalmente, se presentan las conclusiones que se hallaron en el desarrollo del presente documento.

\section{MARCO REFERENCIAL}

Existen diferentes formas de transportar materia prima de un sitio a otro ya sea de forma manual, semiautomática o automática. Una de estas maneras es utilizando un vehículo de guiado autónomo definido como "robot móvil controlado por un computador, utilizado para el transporte de material" (Echeverri 2012) [13], (Vis 2006) [14]. Las principales áreas de aplicación de estos dispositivos se encuentran en la intralogística, concretamente en la organización, control, ejecución y optimización de flujos internos de bienes y materiales, canales de información y transferencia de mercancías en la industria, el comercio y las instituciones públicas (Gunter 2015) [15].

Hay que tener en cuenta en el contexto de la Industria 4.0 , se pueden encontrar múltiples desafíos. En primer lugar, las implementaciones actuales de los AGV generalmente no son capaces de adaptarse al entorno de la industria donde se ejecutan sistemas de manufactura flexible (FMS). En segundo lugar, la visibilidad en tiempo real de la ubicación, la trayectoria y las colisiones apenas se informan (Theunissen 2018) [16]. Por lo anterior, se hace necesario que los AGV tengan la capacidad de compartir información en todo momento del estado actual, o por lo menos, como lo requiera el proceso, de manera que se pueda consultar a través de una interfaz amigable y de diferentes dispositivos como teléfonos celulares y computadoras personales y esté disponible para los usuarios interesados $y / 0$ dueños de un proceso productivo (ver figura 2).
Adicionalmente, en el marco de la Industria 4.0 este tipo de robots, han venido siendo adaptados bajo este concepto de ciberindustria, por lo que se hace necesario diferentes modificaciones que tengan en cuenta variables como peso, carga útil, velocidad de movimiento 0 incluso más cantidad o tecnologías de sensores que lleven a los fabricantes de AGV's a diseñarlos y construirlos bajo cierta normatividad. Para ello hay normas como la ISO 10218, la TS-15066 publicada en el año 2016 el cual ayuda al desarrollador a verificar y gestionar el riesgo en la interacción humano-robot (Chen 2017) [17].

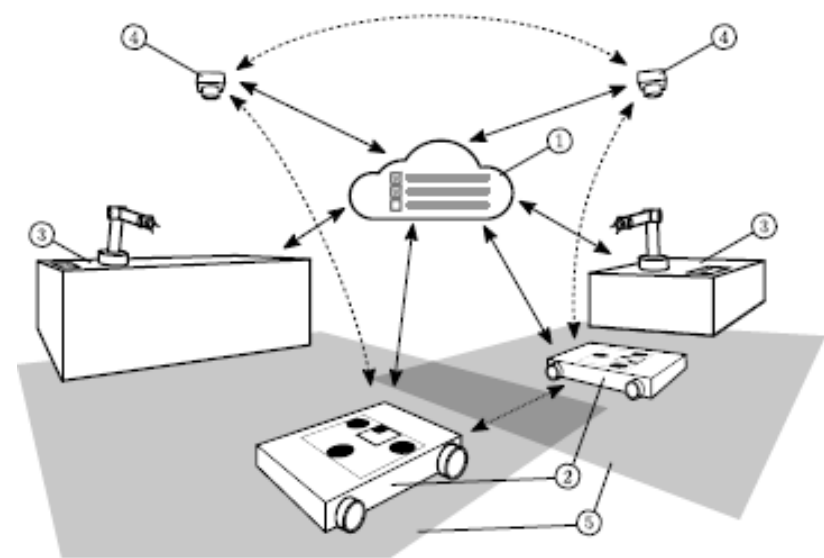

Figura 2. AGV en el contexto de la Industria 4.0. (2) AGV's transmitiendo información a sensores (4) y a

la nube (1), (3) FMS compartiendo datos con la nube (Van 2018) [18].

Es importante mencionar también que cada AGV, de acuerdo al ambiente donde se va a desempeñar, está construido específicamente para una tarea particular. En otras palabras, es importante tener en cuenta los espacios y las características de los productos que se van a transportar de un sitio a otro para un diseño y construcción adecuada de cada robot a implementar. Hay que resaltar que también se debe pensar más allá al momento de realizar dicho diseño, por ejemplo, si se quiere construir un vehículo que cumpla con especificaciones para conectarse con otro tipo de dispositivos o bases de datos (Industria 4.0), así el proceso no se encuentre preparado para ello, pensando en que a futuro si se pueda hacer. Un ejemplo de lo mencionado anteriormente se puede observar en la figura 3. 
Debido a este amplio margen de aplicaciones, se debe tener en cuenta los siguientes aspectos: número de vehículos que va a comprender el sistema, diseño de trayectorias, adaptabilidad al entorno de trabajo, tipo y cantidad de material a transportar teniendo en cuenta el cómo, cuándo y dónde realizará la carga y descarga de este, manejo de las baterías de los vehículos y cuáles serán los sistemas de seguridad que evitarán las posibles colisiones que afecten la integridad del sistema y por ende a él o los usuarios con quienes va a interactuar (Vis 2006) [14] , (Zapata 2015) [19].

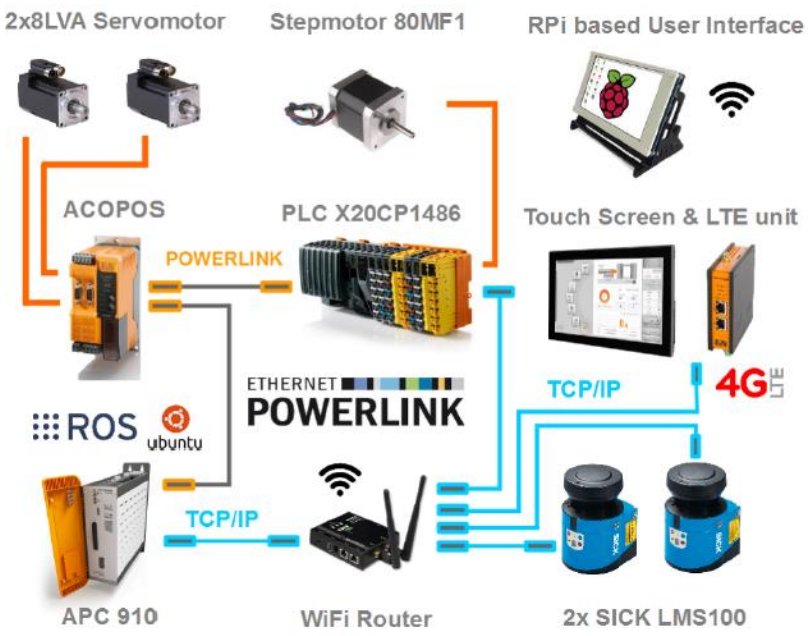

Figura 3. Algunos componentes mecánicos, electrónicos y de comunicación que usa un AGV y lo prepara para la Industria 4.0 (Bacik 2017) [20].

De ahí que autores como (Vis 2006) [14] y (Kumar Das 2016) [21] se preocupen por realizar un diseño previo del AGV de acuerdo al sitio de aplicación, no sin antes hacer una definición previa de algunos términos que son estándar para cualquier vehículo a desarrollar. Los términos que define a groso modo son los siguientes:

- Trayectoria: Se define como las rutas guiadas fijas en las que los vehículos pueden viajar a los diversos puntos de recolección y entrega de cargas. Se debe tener en cuenta que estas pueden ser físicas o virtuales (Walenta 2017) [22].

- Gestión de tráfico: Reglas necesarias para evitar colisiones y situaciones de estancamiento en las que dos o más vehículos están bloqueados por completo.
- Ubicación de los puntos de recolección y entrega: Los puntos de recogida y entrega conectan la red AGV, por ejemplo, máquinas, estaciones de carga, estaciones de inspección y lugares de almacenamiento.

- Requerimientos del vehículo: Son las características que debe tener el AGV mínimamente para encajar de forma aceptable en el sistema (tamaño, forma, sensores, sistema de guiado, programación, entre otros).

- Gestión del AGV: Son las normativas de control que satisfacen las demandas de transporte lo más rápido posible y sin que haya conflictos entre vehículos. Por lo tanto, al menos las siguientes actividades deben ser realizadas por un controlador del sistema: Despacho del vehículo, rutas para el vehículo, programación del vehículo y posicionamiento de vehículos inactivos.

- Aspectos tecnológicos: Son los aspectos que al modelar los AGV, se deben tener en cuenta ya que son las características específicas de los sistemas automatizados. Básicamente son dos, primero, los vehículos sin conductor, generalmente usan baterías y deben cargarse 0 cambiarse y segundo, el tiempo requerido para realizar estas operaciones, los cuales puede afectar el rendimiento del sistema.

\section{ESTADO DEL ARTE}

El objetivo de esta revisión es orientar al lector en algunos criterios para la implementación de AGVs, de acuerdo a unas características específicas del sitio donde se desea operar el vehículo. Para lograrlo, se hizo una búsqueda de cada documento de acuerdo a cuatro aspectos que se consideran importantes para realizar una implementación de este tipo de tecnología. En primer lugar la identificación del sitio, segundo, la aplicación del AGV en diferentes entornos, en donde hay que tener en cuenta temas logísticos, constructivos e infraestructura, en relación a cableado eléctrico, contratación de terceros para dichos movimientos y adecuación del lugar para disposición de maquinaria, no es tan sencillo mover dichos 
equipos para la implementación de un sistema de transporte autónomo. En tercer lugar, control del AGV, en donde es importante mencionar algunas técnicas de control empleadas en el desarrollo de este tipo de tecnología y finalmente en cuarto lugar, el entorno de software para la programación de AGV's con diferentes aplicaciones industriales, haciéndose particular énfasis en ROS para la programación de diferentes tipos de robot como un software estándar que facilita, agiliza y se está fortaleciendo globalmente para el desarrollo de nuevas tecnologías en el campo de la robótica (Garber 2013) [23].

\subsubsection{Identificación de instalaciones industriales y planificación de rutas.}

Para cualquier tipo de proyecto que se quiera ejecutar en un entorno industrial, generalmente es muy importante hacer un reconocimiento de las instalaciones para así determinar si es viable o no la implementación de la solución al problema planteado. Sin embargo, autores como (Drira 2007) [24] van un poco más allá de no solo evaluar las instalaciones industriales, sino de diseñar a priori la distribución de la maquinaria, rutas de acceso, iluminación del recinto, entre otros. Básicamente este autor parte del diseño de las instalaciones, debido a que un mal diseño puede impactar considerablemente en el rendimiento del flujo de producción. Por lo anterior, propone un marco de trabajo general donde se describen criterios para el diseño de la disposición y caracterización de los sistemas de manufactura, teniendo en cuenta consideraciones estáticas y dinámicas, representaciones continuas y discretas, formulación de problemas y enfoque de resoluciones. Sin embargo, hay que considerar que algunos diseños se centran en determinar solo la posición espacial de las instalaciones, dejándose a un lado la elección del tipo de recurso de fabricación o transporte, diseño de las celdas de trabajo, entre otros.

Teniendo lo anterior en mente, la metodología de diseño de disposición de instalaciones es uno de los aspectos principales a tratar para un funcionamiento eficiente y económico de un sistema automatizado de vehículos guiados (Gutta 2018) [25]. Según este autor, para los fabricantes de EE. UU se reduce entre un $10 \%$ y un $30 \%$ del costo total de manejo de materiales debido a un diseño eficiente de las instalaciones. El diseño de la disposición de las instalaciones se clasifica en diseño de la infraestructura, puntos de recolección y entrega, y el diseño de la trayectoria de flujo. Por tal razón, (Gutta 2018) [25] propone dividir en el diseño el espacio de trabajo en un conjunto de polígonos complejos conocidos como celdas, logrando así un funcionamiento secuencial adecuado de un sistema de fabricación debido a que el diseño se combina con el sistema de flujo de materiales. Sin embargo, dada la naturaleza única de los sistemas de fabricación, las tecnologías en evolución y el hecho de que no siempre se cuente con la oportunidad de diseñar las instalaciones de acuerdo a las necesidades del flujo productivo, se deben desarrollar técnicas lo suficientemente robustas que permitan que el AGV se adapte al sitio donde transite.

Siguiendo con la línea anterior, hay autores que también identifican el diseño de las instalaciones industriales como uno de los principales cuellos de botella en el tiempo de implementación de este tipo de vehículos. Este es el caso de (Beinschob 2017) [26], el cual argumenta que el largo tiempo de ejecución tiene varias razones: en las instalaciones de planta de última generación, la infraestructura designada sigue siendo necesaria para la localización; el proceso de mapeo requiere de personal altamente calificado y, en cualquier caso, la información de posición no disponible o inapropiada de los puntos de entrega para los productos, debe ser corregido en sitio. En total, la configuración de una planta para estar lista para la operación de un AGV está tomando varios meses, vinculando a técnicos altamente calificados e involucrando procedimientos in situ muy costosos y que requieren mucho tiempo. Por lo tanto, (Beinschob 2017) [26] presenta un enfoque para el despliegue de un AGV que tiene como objetivo reducir drásticamente el tiempo, el costo y el personal involucrado. En primer lugar, propone el empleo de una novedosa tecnología de escaneo láser SICK 3D preparada para la industria con el fin de construir una representación virtual precisa y coherente de todo el entorno del almacén. Mediante la utilización de una segmentación y un procesamiento adecuado, se genera un mapa semántico que contiene posiciones georreferenciadas en 3D, así como un mapa de localización 2D que elimina la necesidad de una solución dedicada al mapeo 2D. Por ejemplo en la figura 4 se presenta un mapa $3 \mathrm{D}$ con gráfico de características de un almacén industrial, la línea roja es la trayectoria estimada, las líneas amarillas 
son los bordes entre la posición actual y los puntos de características relacionados, los grises son puntos de escaneo. Las líneas de cuadrícula gris claro marcan distancias de $50 \mathrm{~m}$. En segundo lugar, el mapa semántico proporciona un mapa de espacio libre que se utiliza como base para la creación automática de la ruta con el fin de lograr un flujo óptimo. Aunque la intervención humana sigue siendo necesaria en varios pasos, incluido el diseño de la trayectoria para el mapeo, la conducción del vehículo de mapeo, la verificación de la consistencia del mapa, correcciones manuales ocasionales en el gráfico y la definición de regiones de interés para la extracción del elemento del mapa semántico. Por lo tanto, la metodología propuesta por (Beinschob 2017) [26] se define como semi-automatizada.

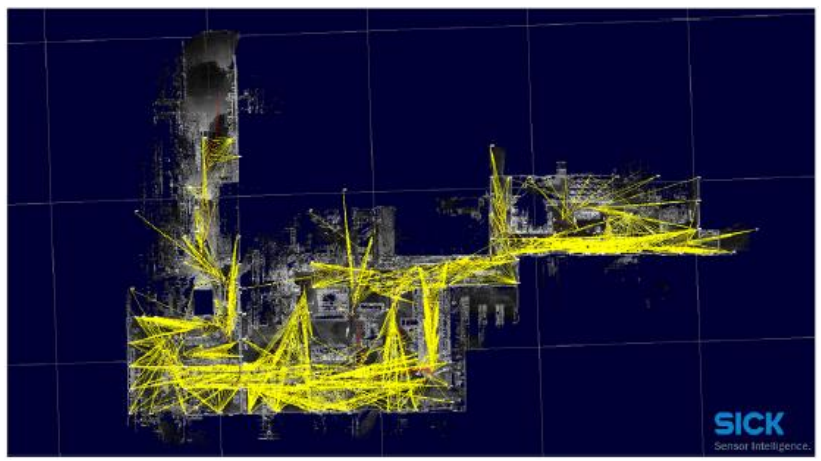

Figura 4. Mapa 3D (Beinschob 2017) [26].

Otros autores proponen diversas soluciones que ayudan a implementar un sistema de vehículos autónomos en zonas o procesos donde ya no es posible diseñar la infraestructura o el local, debido a que se diseñó a priori al proyecto tecnológico a ejecutar. Dadas estas circunstancias, algunos hallazgos significativos han sido seleccionar un modelo de navegación que se ajuste fácilmente no solo al vehículo sino también al ambiente de trabajo donde va a operar. Algunos estudios primarios muestran la viabilidad y la practicidad del uso de RFID (por sus siglas en inglés: Radio Frequency Identification) para el posicionamiento de AGV's en industrias de manufactura típica, usando lectores y etiquetas en el entorno del proceso a fin de facilitar la logística automática a través de un controlador remoto (Lu 2017) [27]. Otra manera de utilizar este tipo de tecnología en los AGV es implementándolo como un sistema de seguimiento de posición en interiores basado en un algoritmo de seguimiento preciso a través de puntos de referencias cercanos al vehículo (Saab 2011) [28], es clave el enfoque de localización y navegación que debe ser altamente flexible para tratar aplicaciones industriales complejas, y más si se desea aplicar el RFID, como etiqueta o antena (Lu 2018) [29], donde la selección de dicho elemento es de suma importancia, en primer lugar, si se elige etiquetas como medio de navegación, el área de lectura cobra cierta relevancia debido a que el lector recibirá más o menos radiación o energía reflejada según la posición del elemento pasivo. En segundo lugar, si se elige la antena como medio de navegación, se debe obtener la función direccional y el ancho del haz para que el sistema funcione en un entorno estable. Pese a que esta tecnología ayuda en gran medida a la navegación y el posicionamiento del vehículo en la planta, la velocidad de conducción, el tamaño de AGV, el entorno, la capacidad de cálculo y muchos otros factores pueden influir en la precisión de posicionamiento del sistema de RFID (Lu 2017) [27] - (Lu 2018) [29].

Por otro lado, autores como (Hartmann 2014) [30] desarrollan un sistema de cronogramas para AGV's dentro de diferentes ambientes de sistemas de manufactura flexible (FMS por sus siglas en inglés: Flexible Manufacturing System) usando algoritmos genéticos (GA por sus siglas en inglés: Genetic Algorith). Básicamente cuando se ingresa un cromosoma, el algoritmo genético trabaja sobre él y produce la misma cantidad de cadenas de desconexión, adicionalmente, el número de iteraciones tiene lugar hasta que se obtiene la solución óptima. De ahí que se puedan apreciar varias ventajas inherentes como la posibilidad de determinar el procesamiento de tiempo para el AGV, tiempo de viaje de cada trayecto, tiempo de finalización de la operación y tiempo de finalización de trabajo.

En (Kang 2013) [31] se realiza una aplicación de un método de visión por computadora para adquirir información básica y así controlar un grupo de AGV's. En primer lugar, se diseñan unos marcadores con significados especiales (como "Ir recto", "Giro a la izquierda", "Giro a la derecha" y "Parar"). En segundo lugar, usa una cámara para capturar los marcadores, usando la función HOG (Por sus siglas en inglés: Histograms of Oriented Gradients) y clasifica los marcadores con una máquina de soporte vectorial (SVM por sus siglas en inglés: Support Vector Machines). Después calcula la distancia y el ángulo de desviación desde los marcadores hasta el AGV. Finalmente, se envía la información al AGV controlando los movimientos 
del mismo. Se debe tener en cuenta que la cámara debe tener una buena resolución y adicionalmente la intensidad de luz juega un papel fundamental en el reconocimiento de los marcadores, debido a que en un lugar poco iluminado se puede dar errores de navegación del vehículo (Kang 2013) [31].

Finalmente, en (Valencia-Hernández 2017) [39] se presenta un estudio donde se aplica realidad aumentada para representar obstáculos virtuales en la navegación real de un robot móvil. Mediante este sistema se podría hacer pruebas en plantas de producción, almacenes logísticos 0 bodegas industriales de la implementación de AGVs sin tener la totalidad de los equipos reales en el sitio, ya sea porque no se han adquirido, o se están trasladando desde otro sitio, con lo cual no se pierde tiempo esperando a tenerlos todos, y se podrían hacer pruebas de identificación de instalaciones industriales, planificación de rutas y navegación. También puede ser una buena herramienta para probar diversas fases del diseño de las instalaciones nuevas, antes de que estén completamente terminadas.

\subsubsection{Implementación del AGV en diferentes entornos de trabajo.}

Proporcionar soluciones para el transporte de materia prima dentro de recintos cerrados no es el único beneficio que se obtiene al desarrollar un proyecto con este tipo de característica. Es de notarse también que este tipo de tecnologías tiene un gran margen de aplicabilidad en otras áreas, como por ejemplo en el sector urbano. Este es el caso de (Osman 2017) [32] el cual aborda la confiabilidad operativa y la seguridad de los vehículos de guiado autónomo a lo largo de las carreteras, en donde se requiere una integración muy estrecha entre la detección ambiental y el control de la dinámica del vehículo. Esto se basa en determinar los límites relevantes en un carril, utilizando una cámara convencional y una estrategia de control lateral y longitudinal limitada para un AGV cuya dinámica se describe mediante un modelo no lineal con dos entradas, el torque del eje trasero y el torque de dirección angular. El objetivo es controlar el AGV para seguir una trayectoria deseada mientras se evita la salida del carril. Para este propósito, se utiliza un algoritmo basado en visión para determinar los límites de los carriles de la carretera con respecto al vehículo, luego se genera una trayectoria de referencia dentro de estos límites basados en las funciones básicas de Hermite. Las entradas de control para el AGV se diseñan posteriormente de tal forma que rastrea la trayectoria dada mientras su posición permanece en los límites del carril. Para lograr esto se abordan dos enfoques, uno basado en características y el otro en el modelo. El primero tradicionalmente utiliza la segmentación de imágenes para detectar los carriles. Sin embargo, este método no impone restricciones en las formas del carril, lo que puede causar una detección de carril defectuosa debido a la oclusión o al ruido. El segundo método utilizado por (Osman 2017) [32], representa el carril utilizando modelos matemáticos. Otra aplicación de los AGV es en el sector de la salud, más propiamente en las clínicas y hospitales, tal como el que se observa en la figura 5 (Bacik 2017) [20]. Actualmente la demanda de robots móviles y su uso en hospitales se ha incrementado debido a los cambios en las tendencias demográficas y el control de los costos médicos. Para instalaciones de salud, estos sistemas automáticos están diseñados específicamente para manejar materiales granulados, medicamentos de farmacia, muestras de laboratorios, suministro central y transporte de alimentos, platos sucios, ropa de cama, desechos (biológicos, reciclables), instrumentos biomédicos, etc. (Pedan 2017) [33] ha visualizado que automatizar estos suministros, permite la transferencia de recursos humanos a otros departamentos 0 actividades. Los sistemas automatizados funcionan las 24 horas del día, los 7 días de la semana. La solución automatizada puede agilizar el flujo de tráfico de material en el hospital, controlar los costos y reducir la carga de trabajo. La instalación operativa del hospital debe cumplir algunos requisitos importantes, tales: seguridad y calidad clínica, productividad, fácil de usar por pacientes, familias y personal de la clínica, servicio de salud excelente y utilización efectiva de la tecnología como información y manejo del paciente (Pedan 2017) [33]. Estos requisitos y el uso de sistemas logísticos modernos afectan significativamente el funcionamiento de toda la instalación y su economía, mejoran la calidad de la atención del paciente y aumentan su seguridad.

Sin embargo, (Pedan 2017) [33] también menciona que la evaluación económica realizada indica que los AGV actualmente no son económicos y solo son asequibles para instalaciones más grandes que se administran con fines de lucro. Un trabajo similar presenta (Acosta 2015) [34], el cual menciona la importancia de mejorar el flujo de trabajo del 
personal de salud. Por esta razón propone el despliegue de un vehículo automatizado que ayude a la productividad de las tareas logísticas dentro de un hospital, como la recolección y entrega de productos, así el personal puede enfocarse en las tareas necesarias para servir mejor a la comunidad. En ese orden de ideas (Acosta 2015) [34] propone desarrollar estaciones internas en donde primeramente se crea un mapa de cada piso del local y posteriormente se indica donde quedará ubicada cada estación, adicionalmente es posible indicar lugares de interés, puntos de recolección y entrega, para que el AGV navegue de forma autónoma dentro del recinto (ver figura 6). Esto reduce el costo adicional dibujando las líneas o trayectos en el suelo. Adicionalmente, el AGV es capaz de interactuar o navegar de forma segura con los usuarios del hospital mientras transporta los objetos requeridos o solicitados. Hay que resaltar también que el prototipo desarrollado por este autor es bastante robusto a nivel de software, debido a la capacidad de seguir trayectorias y/o seleccionar la mejor ruta gracias al mapa precargado que posee en memoria, como se muestra en la figura 7. Esto facilita al robot conocer a priori los puntos de recolección y entrega del material a transportar. Cabe mencionar que esto es posible debido al tipo de software que utiliza, en este caso ROS, al ejecutarse en Linux, permite un procesamiento más alto de las operaciones internas del robot, en este caso lectura y procesamiento de mapas y presenta la característica de abstracción del hardware el robot para así aprovechar de manera más óptima los sensores y actuadores instalados en él.

Particularmente para el robot desarrollado por este autor, para recorrer los mapas precargados adecuadamente, se necesita hacer uso de un IMU (por sus siglas en inglés: Inertial Measurement Unit) (Barnea 2011) [35], para la navegación y localización de un sistema robótico autónomo, ayudando así a conocer la posición, orientación y desplazamiento del robot dentro del mapa. Por otra parte, este entorno de software, permite realizar simulaciones del robot facilitando de esta manera el desarrollo del prototipo y acelera los tiempos de prueba debido a que se puede realizar un análisis a priori antes de la implementación en sitio. De igual manera, en la sección 3.1.4 se abordará más a fondo este tema.

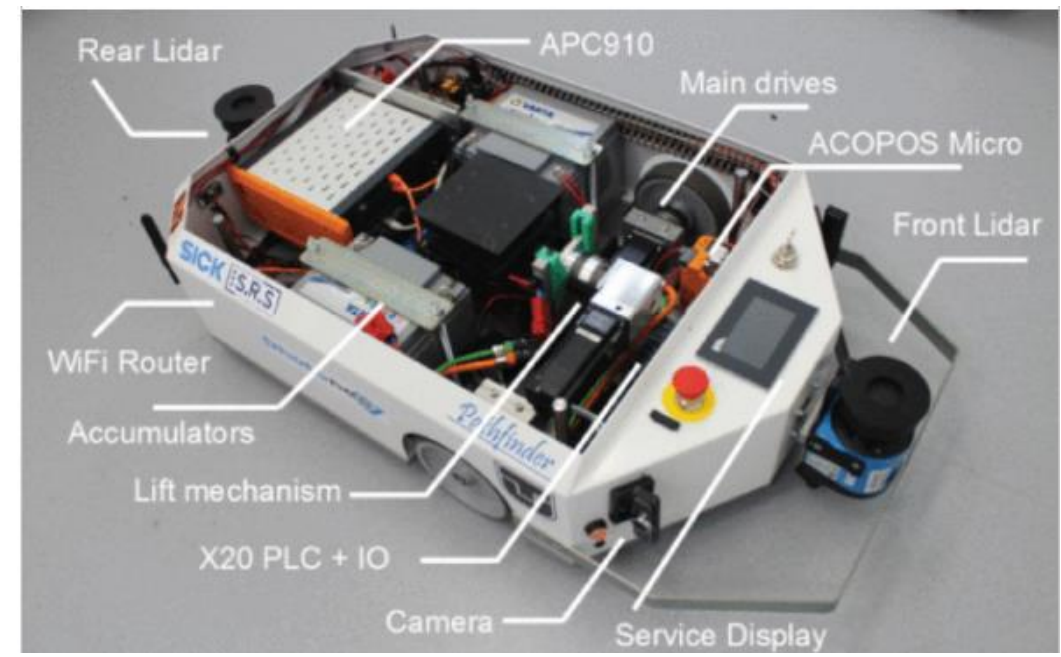

Figura 5. AGV's para instalaciones de salud (Basik, 2017) [20].

Por otra parte, existe otro modelo de negocio público que también se puede beneficiar con este tipo de tecnología. Este es el caso de las bibliotecas. Para este tipo de lugares es interesante debido a que se pueden evidenciar diariamente diversos procesos, como la ubicación y recolección de libros, el cual usualmente es realizado por personas. De acuerdo a lo anterior, (Sepúlveda 2014) [36] propone un modelo de AGV el cual radica en el beneficio de explotar la funcionalidad de un robot en trabajos que son pesados y repetitivos. Dicho vehículo automatiza el proceso de caracterización, categorización, referenciación y ubicación de libros en un ambiente con condiciones controladas. Aunque, la infraestructura donde se implemente el vehículo debe igualmente estarlo por ejemplo con un sistema de almacenamiento automático de libros, cada uno referenciado por un 
identificador que reconoce la memoria del robot (Sepúlveda 2014) [36].
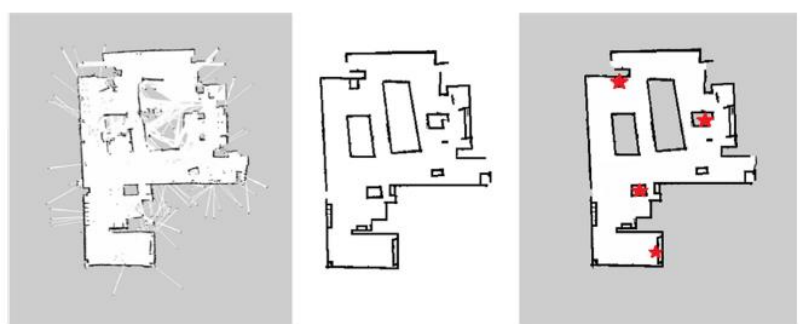

Figura 6. Mapa del laboratorio del autor (Acosta 2015) [34].

Finalmente, el área de aplicación donde actualmente se usan con mayor frecuencia los AGV's son en el sector industrial. De acuerdo a un estudio realizado por (Sabattini 2017) [37], en las plantas de fabricación modernas, la automatización es ampliamente adoptada en las fases de producción, lo que conduce a un alto nivel de productividad y eficiencia. Sin embargo, generalmente no se logra el mismo nivel de automatización en logística, ya que es realizado por operadores humanos y vehículos de accionamiento manual. De hecho, aunque los AGV se han utilizado durante algunas décadas para el transporte de mercancías en entornos industriales (Gunter 2015) [15], todavía no representan una solución generalizada y se aplican solo en escenarios específicos. Un ejemplo es el sistema Kiva (Wurman 2008) [38], donde los AGV son utilizados como estantes de almacenamiento móviles que pueden ser levantados por pequeños robots autónomos. Al llevar el producto al trabajador, la productividad se incrementa en un factor de dos o más, a la vez que mejora otros aspectos. Si bien esta solución es muy efectiva para este tipo de aplicaciones, vale la pena señalar que no se puede aplicar directamente a la manipulación de materiales en fábricas genéricas. Lo que hace que este sistema sea único es que los AGV se mueven en un entorno restringido, donde otras entidades no están permitidas (Sabattini 2017) [37], (Wurman 2008) [38]. Esto hace que el sistema no sea aplicable a entornos mixtos compartidos por operadores humanos, vehículos de accionamiento manual y AGV.

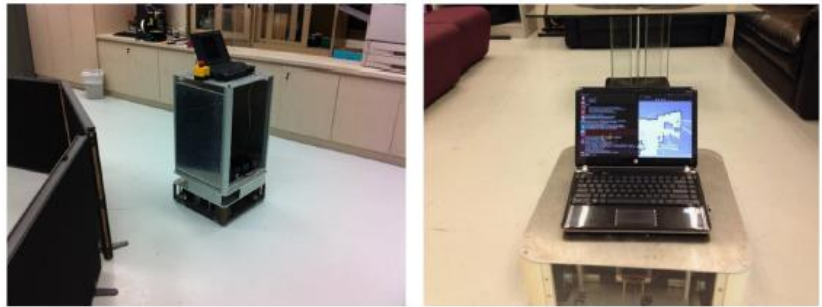

Figura 7. AGV durante la navegación en diferentes escenarios para la construcción de mapas (Acosta 2015) [34]

\subsubsection{Algunos de métodos de control del AGV en recintos cerrados.}

Existen diversas técnicas de control que ayudan a los AGV a cumplir con las tareas programadas dentro de una planta de producción o cualquier otro espacio en donde se soliciten. Muchas de estas técnicas son aplicadas de acuerdo a la especificidad del proyecto o la necesidad de resolver una situación con una técnica específica. Este es el caso de (Silvirianti 2017) [12] el cual busca la estabilidad del movimiento de un AGV debido a que este afecta directamente la seguridad de los productos entregados. Este autor argumenta que la variación de la carga durante el proceso de entrega es una de las causas del movimiento de inestabilidad de los vehículos. Por tal motivo, implementa un control de velocidad sin importar la variación de la carga utilizando un sistema de inferencia difusa integrado con el método PID. La entrada del sistema proviene de los sensores de velocidad e inclinación cerca de las ruedas del AGV. Esta información es luego procesada por el sistema de inferencia difusa, cuyo resultado son datos sobre la velocidad del AGV y los valores de los parámetros para el controlador PID. Adicionalmente utiliza sensores de ultrasonido para evasión de los obstáculos, encoders en las ruedas que retroalimentan hacia el sistema de control y RFID para la navegación del vehículo en sitio. En definitiva, (Silvirianti 2017) [12] logra una velocidad constante de $10 \mathrm{~m} / \mathrm{min}$ independiente de la variación de la carga en el AGV.

Otros controles están propuestos en (Parikh 2018) [40], donde se propone una comparación entre técnicas de control para un AGV que se traslada a velocidad constante, logrando esto con la implementación de un controlador difuso y un controlador PID, realizando la realimentación por medio de encoders adaptados a las ruedas del 
vehículo. Adicionalmente, utiliza los algoritmos de Ziegler-Nichols para sintonizar el controlador PID y las técnicas de Mamdani para el controlador difuso. Posteriormente, para probar todo el modelo de la planta y del control, utiliza Matlab/Simulink y las librerías de lógica difusa, llevando la información recolectada a un arduino quien es realimentado con las señales del encoder y del control de velocidad de los motores. Finalmente, (Parikh 2018) [40] concluye que el error en estado estable, el sobre impulso y el tiempo de subida del sistema, en el controlador PID son mayores que para el controlador de lógica difusa, brindando este último más estabilidad y precisión al sistema.

Una forma interesante de utilizar la lógica difusa es en la alineación o formación de vehículos autónomos, donde un grupo de móviles siguen a un líder. Se presenta entonces un sistema de control de formación de robots, en este caso AGV's, basados en visión, el cual utilizan un controlador de lógica difusa y un filtro de Kalman extendido (Rusdinar 2012) [41]. Según (Rusdinar 2012) [41], estos sistemas presentan problemas que afectan los controles de formación con el controlador de lógica difusa y los robots basados en visión, los cuales son: la selección de la función de pertenencia para obtener el control de sistema difuso óptimo y el ruido proveniente del procesamiento de la cámara, los cuales cambian la posición de la vista de referencia. Para manejar este tipo de situaciones, (Rusdinar 2012) [41] propone un controlador difuso equipado con una función dinámica de membresía de salida que controla la velocidad de las ruedas del robot para manejar la posición de mantenimiento en la formación. La función de membresía de salida cambia con el tiempo en función de los cambios en las entradas. Por otra parte, los ruidos que aparecen en el procesamiento de la imagen cambian las posiciones del punto objetivo virtual que maneja el filtro de Kalman extendido por lo cual las posiciones del objetivo virtual se establecen para definir las formaciones. Adicionalmente, las posiciones del punto objetivo virtual se pueden cambiar en cualquier momento de acuerdo con la formación deseada. Finalmente, estos algoritmos han sido validados a través de la simulación, confirmando que los robots seguidores alcanzan su punto objetivo en poco tiempo y pueden mantener su posición en la formación, aunque el ruido cambia las posiciones del punto objetivo (Rusdinar 2012) [41].
Es importante reconocer entonces que con algunas herramientas informáticas se hace más sencillo y efectivo determinar los parámetros óptimos de un control PID, un controlador difuso o uno PID difuso. En la actualidad, se implementan cada vez más aplicaciones para los controladores PID difusos que tienen una configuración mucho más flexible que sus análogos lineales. Los métodos para determinar los parámetros óptimos de los controladores PID difusos son bastante complejos y consumen mucho tiempo, lo que restringe significativamente su aplicación (Kudinov 2017) [42]. Debido a esto, (Kudinov 2017) [42] propone un método simple de controladores, particularmente el PID difuso, con el uso de Matlab, adicionándoles ciertas disposiciones del método de linealización difusa, dividiendo a su vez este procedimiento en tres pasos: Identificación del controlador difuso, estimación de los coeficientes del controlador difuso y finalmente, construcción de un sistema de control digital.

Cuando las aplicaciones desarrolladas para el AGV son a altas velocidades, se presentan problemas de fuerzas de fricción, vibraciones y deslizamiento entre las ruedas y el terreno (Ahmed 2015) [43]. En este orden de ideas, (Ahmed 2015) [43] hace referencia a los problemas de seguimiento de trayectos, los cuales en la mayoría de ocasiones son inciertos, y crean cierta borrosidad en la entrega de información al sistema. Es por esto que, como los modelos determinísticos no son suficientes, dicho autor propone un controlador PID neuronal difuso para compensar la fricción y los efectos de perturbaciones externas durante el seguimiento de trayectorias de un robot móvil no holonómico, en donde los parámetros del control adaptativo se actualizan de acuerdo con un algoritmo de aprendizaje en línea.

Una forma para desarrollar la inteligencia de los AGV para el seguimiento de trayectos en complejos industriales 0 edificios y con autorización para ingresar a lugares restringidos, es a través del seguimiento de líneas usando sensores de color y RFID para la navegación en el medio, como se propone en (Nugraha 2015) [44], donde se plantea un robot seguidor de línea diseñado para detectar diferentes colores de línea representadas en diferentes rutas. Este sistema utiliza un microcontrolador que implementa lógica difusa, particularmente el método de inferencia de Mamdani. Adicionalmente para detectar la línea, el vehículo está equipado con un sensor de color y un 
sistema de identificación y autorización basado en RFID. Cabe aclarar que a pesar que las rutas están trazadas en el suelo facilitando el recorrido de trayectos realizados por el robot, para cambios de colores muy oscuros en la superficie del suelo, el sistema necesita ser adaptado para así reconocer estos cambios, evitando que el vehículo se pierda en el trayecto (Nugraha 2015) [44]. Es importante mencionar que al implementar este tipo de tecnología en los AGV se está contribuyendo para que el robot tenga la capacidad de compartir información con otras máquinas y con bases de datos en la nube, haciendo posible capturar los datos en tiempo real para la logística automática en una fábrica inteligente, lo que lo hace importante en la industria 4.0 (Zou 2018) [45]. Según este autor, utilizando esta tecnología se puede lograr obtener un mapa virtual de la planta, o por lo menos de los puntos más importantes y de interés para el usuario final, debido a que brinda la capacidad para ayudar en la toma de decisiones, y esto se facilita debido a que al integrar estas etiquetas en las rutas ayudan a identificar la ubicación para lograr la logística automática, como se muestra en la figura 8.

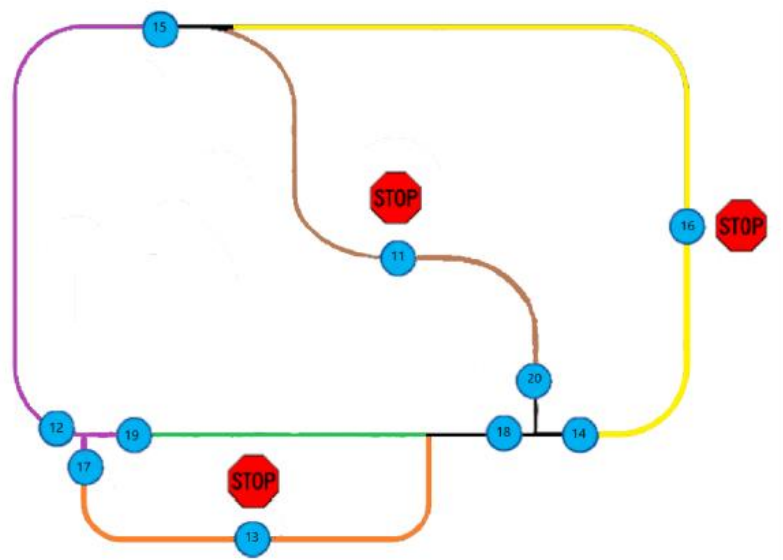

Figura 8. Diseño de rutas con etiquetas RFID, estaciones de paros y en color se muestran las trayectorias a recorrer (Zou 2018) [45]

Basado en el modelo cinemático, (Bui 2013) [46] presenta un controlador de seguimiento para un AGV, que rastrea una trayectoria de referencia, usando el sensor laser NAV200. Inicialmente el AGV se mueve manualmente para rastrear la trayectoria de referencia. A partir de ese momento, las coordenadas obtenidas del sensor laser de la posición del AGV son almacenadas en memoria. Luego estos datos se utilizan para ayudar al AGV a trasladarse repetidamente por esta trayectoria en el futuro. Para el diseño del controlador se usó la técnica no lineal de Lyapunov, verificando así la estabilidad y a su vez, proporcionando robustez para las perturbaciones generadas por la carga y el ruido de los sensores, de manera que pueda seguir de forma eficaz la trayectoria. El vehículo consta de un sensor láser NAV200 para detectar la posición del AGV en tiempo real, un controlador basado en una computadora industrial (figura 9), adicionalmente 4 ruedas, dos de ellas pasivas y dos motrices (figura 10). La eficacia del controlador propuesto se prueba mediante simulación y resultados experimentales (Bui 2013) [46].

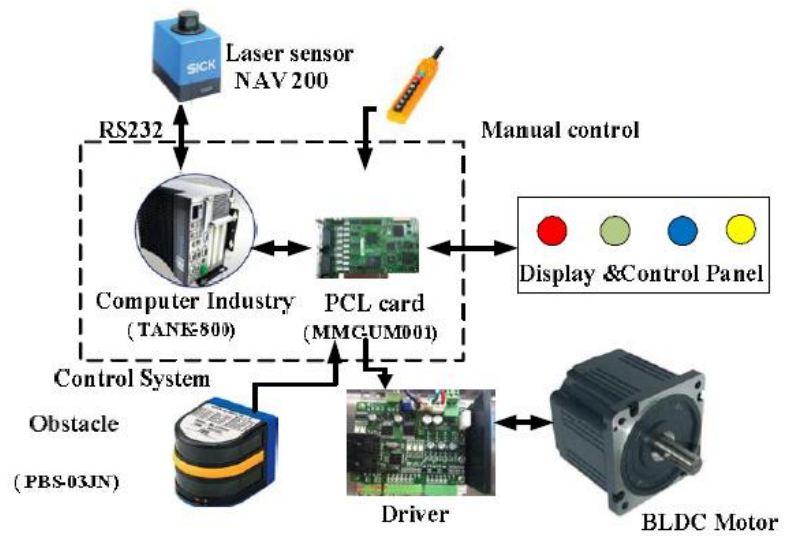

Figura 9. Esquema del sistema de control para el AGV propuesto por (Bui 2013) [46].

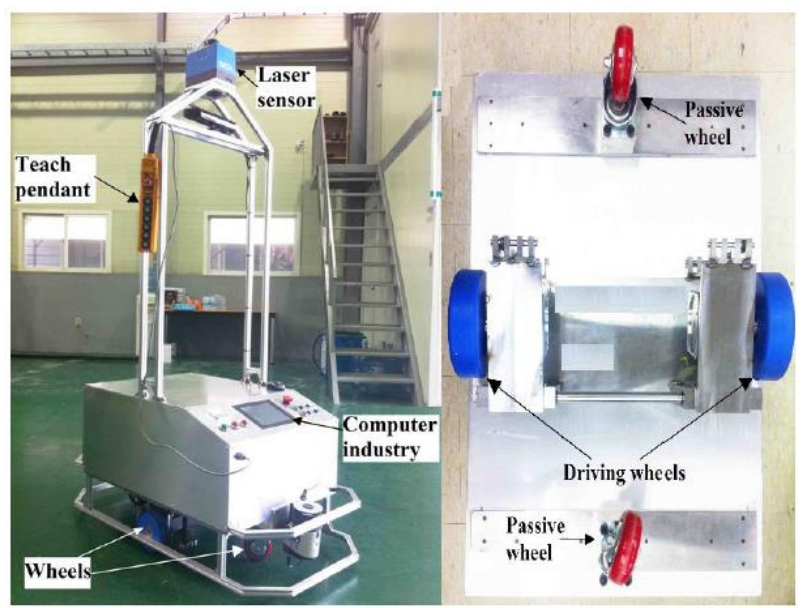

Figura 10. Prototipo de un AGV experimental (Bui 2013) [46]

Por otro lado, (Carlucho 2017) [47] plantea mejoras en las técnicas de control clásico para perfeccionar las respuestas de los sistemas. Dicho autor ha estado desarrollando sistemas expertos e 
inteligentes para controlar muchos sistemas tecnológicos, incluidos los robots móviles. Por otra parte, el controlador PID es una estrategia de control ampliamente usada en muchas tareas de ingeniería de control, contribuyendo con diferentes métodos de ajuste para obtener las ganancias en condiciones de operación específicas, pero en ocasiones, los sistemas no se conocen completamente y las condiciones operativas son variables y desconocidas previamente, en donde las técnicas clásicas no son del todo adecuadas para la sintonización de un PID (Carlucho 2017) [47]. De lo anterior, (Carlucho 2017) [47] propone una estrategia incremental de Q-learning para el control PID adaptativo para mejorar la eficiencia del aprendizaje, definiendo una memoria temporal en el proceso. Si bien la memoria permanece invariable, se lleva a cabo un proceso de especialización no uniforme que genera nuevos sub-espacios de aprendizaje limitados, implementando entonces un robot móvil el cual demuestra la aplicabilidad del enfoque propuesto para un ajuste simultáneo en tiempo real de múltiples controladores PID adaptativos para un sistema que opera bajo condiciones variables en un entorno. Sin embargo, el comportamiento de los sistemas de dirección de deslizamiento, como en algunos robots móviles, es complejo para modelar matemáticamente porque la maniobra de giro se realiza deslizando sobre el suelo donde la cantidad de derrape varía con el terreno. La velocidad y la dirección de los sistemas de dirección deslizante dependen mucho entre sí. Cualquier intento de maniobrar el vehículo afecta su velocidad y viceversa. La diferencia de velocidad y/o dirección de rotación en ambos lados hace que el vehículo gire y que se produzca un deslizamiento en esta maniobra (Carlucho 2017) [47].

\subsubsection{Sistema software para la programación de vehículos de guiado autónomo.}

Actualmente existen diferentes lenguajes de programación, y metodologías para programar robots, en este caso en particular AGV's, que suelen ser sistemas complejos que demandan cierto grado de conocimiento en varios campos de la ingeniería debido a que son sistemas que integran diversos componentes de hardware, tanto mecánicos, eléctricos y electrónicos, convergiendo en un equipo que se debe desempeñar satisfactoriamente en un área o lugar de trabajo específico. Para lograr dicho objetivo, se han desarrollado diferentes componentes de software que coordinan todos estos mecanismos físicos. (Hellmund 2016) [48] hace una comparación entre tres sistemas de software para la programación de vehículos autónomos.

El primero de estos sistemas es el KogMo-RTDB (Por sus siglas en inglés: Real-time Database for Cognitive Automobiles), el cual es una arquitectura centralizada que proporciona una asignación de memoria dinámica para objetos de datos, y a su vez la memoria debe asignarse estáticamente dentro de los objetos (Hellmund 2016) [48]. Adicionalmente, este entorno de software posee herramientas para grabar y reproducir objetos de datos. Sin embargo, la manera como es gestionada la memoria puede convertirse en una situación engorrosa en escenarios donde el tamaño de los objetos varía, por ejemplo, en los datos adquiridos por un láser.

Otro sistema software utilizado para los vehículos autónomos es el ADTF (por sus siglas en inglés: Automotive Data and Time-Triggered Framework), cuyo entorno de pruebas y desarrollo es el más utilizado en la actualidad para los sistemas avanzados de asistencia al conductor (ADAS) (Messner 2015) [49], (Hoffmeister 2016) [50]. Este entorno de software está diseñado como un sistema en tiempo real con cadenas de procesos distribuidas y admite el procesamiento de datos síncrono y asíncrono. Por otra parte, es una herramienta extensible mediante módulos personalizados y permite la integración de toolbox en Matlab/Simulink. Hay que tener en cuenta que ADTF es un producto licenciado, razón por la cual es utilizado principalmente en empresas industriales.

Finalmente, se hará una descripción más detallada de ROS (por sus siglas en inglés: Robot Operating System) debido a la robustez, versatilidad e impacto a nivel global (Garber 2013) [23]. ROS es un software de código abierto que permite el diseño de sistemas complejos pero modulares en un entorno informático distribuido. A pesar que ROS significa Sistema Operativo Robótico, realmente no es un sistema operativo en el sentido tradicional de la gestión y programación de procesos; más bien, proporciona una capa de comunicaciones estructurada capaz de abstraer información de diferente hardware por encima del sistema operativo host, como se muestra en la figura 11 (Quigley 2009) [51]. 
Si bien los componentes de ROS son altamente genéricos, el enfoque principal de ROS y su ecosistema se centra en el desarrollo e investigación de robots. Las partes críticas del desempeño están hechas en $\mathrm{C}_{++}$, pero las aplicaciones que operan en una capa superior o de alto nivel pueden ser escritas en $\mathrm{C}++$, Python, Octave y LISP [52]. Algunos conceptos fundamentales que se deben conocer cuando se realiza cualquier implementación de ROS, son los nodos, mensajes, tópicos, servicios y acciones. De acuerdo con (Quigley 2009) [51], los nodos son procesos que realizan cálculos y se ejecutan dentro de la aplicación del robot. En este contexto, un sistema está compuesto generalmente por muchos nodos.

Un nodo ROS se asigna a un solo proceso del sistema operativo, mientras que los nodelets de ROS residen como hilos dentro de un proceso único llamado ROS Nodelet Manager. La comunicación entre los nódulos se acelera mediante las comunicaciones intra-ROS como lo indica el enlace entre las capas ROS de la figura 11. La comunicación entre procesos, por ej. A través de los nodos, se transmite por medio de las capas de red del sistema operativo (Hellmund 2016) [48].

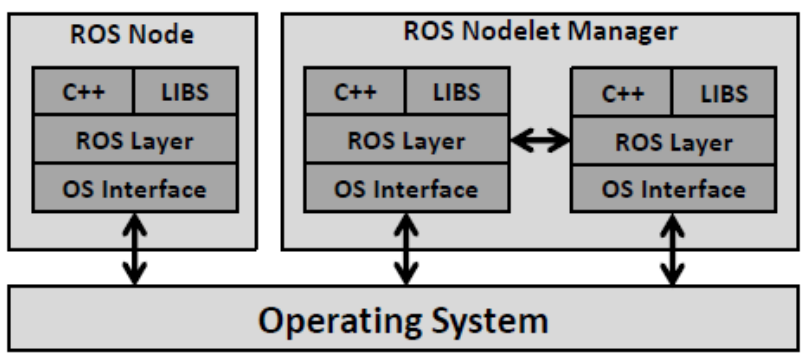

Figura 11. Arquitectura de aplicaciones ROS.

En ese orden de ideas, los nodos se comunican entre sí intercambiando mensajes. Un mensaje es una estructura de datos escrita. Se admiten el tipo primitivo (entero, booleano, punto flotante, etc), al igual que las matrices o estructuras de tipos primitivos y constantes. Una característica de los mensajes es que pueden estar compuestos por otros mensajes y anidados de forma arbitraria. Cuando se transmite un mensaje, este es ruteado vía un sistema de transporte con semántica de publicador/subscriptor y esto se realiza por medio de los tópicos. Los tópicos interconectan los nodos y básicamente cuando un nodo está interesado en cierto tipo de dato, se subscribirá al tópico apropiado y a su vez, este tópico publicará en el o los nodos subscritos a él. En general, los editores y subscriptores no conocen la existencia de los demás. También se debe tener en cuenta que la arquitectura del sistema actual no está capacitada para trabajar en tiempo real debido a la falta de garantías para la ejecución de los nodos y transmisión de prioridad de los mensajes. En otras palabras, ROS no es un sistema operativo en tiempo real, pero permite integrar ROS con el código en tiempo real (Aini 2017) [53]

Finalmente, los servicios y las acciones permiten codificar una funcionalidad específica para el robot. Su principal diferencia es que cuando se llama un servicio, el robot tiene que esperar hasta que este haya terminado antes de hacer otra cosa. Por otra parte, cuando se llama una acción, el robot puede seguir haciendo otra actividad mientras ejecuta dicha acción.

En conclusión, los tres sistemas software presentados muestran ventajas y desventajas los cuales se deben evaluar antes de comenzar a diseñar cualquier robot. En términos generales, estos resultados se pueden resumir en la tabla 1 , en donde se califica de excelente $(++)$, muy bueno $(+)$, bueno $(O)$, poca disponibilidad $(-)$ y casi no disponible (--):

\begin{tabular}{cccc}
\hline Requerimiento & ROS & RTBD & ADFT \\
\hline Modularidad & ++ & ++ & ++ \\
Extensibilidad & ++ & + & + \\
Tiempo - Real & - & + & ++ \\
Simulación & ++ & $\mathbf{0}$ & $\mathbf{0}$ \\
Depuración & ++ & + & ++ \\
Tolerancia a fallos & $\mathbf{0}$ & - & + \\
Seguridad & - & - & $\mathbf{0}$ \\
Usabilidad & ++ & $\mathbf{0}$ & + \\
Soporte & ++ & -- & + \\
\hline
\end{tabular}

Tabla 1. Comparación de sistemas software para robots móviles de acuerdo a (Hellmund 2016) [48].

De acuerdo a la tabla 1, un aspecto interesante que se visualiza para estos tres sistemas software, es la posibilidad de realizar simulaciones de los prototipos que se quiera implementar en algún tipo de planta. Particularmente, ROS presenta herramientas muy poderosas de simulación que tienen como una de sus principales características, 
el permitir trabajar con modelos bajo leyes físicas, lo que garantiza que el robot o proceso simulado presente una alta confiabilidad al momento de implementarlo en el mundo real (figura 12) [52]. Un ejemplo de lo mencionado anteriormente, es el presentado por (Cavanin 2018) [54], quien propone un estudio preliminar sobre la localización de un vehículo autónomo en un entorno industrial, en donde primeramente virtualizó el AGV utilizando ROS, por medio de las herramientas RVIZ y GAZEBO, además de una red de sensores con COOJA, el cual es un simulador de red diseñado específicamente para redes de sensores inalámbricos [55]. Este autor al integrar estos dos sistemas envía al vehículo los datos sobre las intensidades de señal proporcionado por los nodos de la red y seguidamente exporta estos datos en un algoritmo de fusión de sensores como ayudas de medición para la estimación odométrica (Cavanini 2018) [54]. En términos generales, estos dos simuladores se pueden usar para ejecutar algoritmos de seguimiento de rutas y probarlos en un escenario virtual antes de la implementación real.
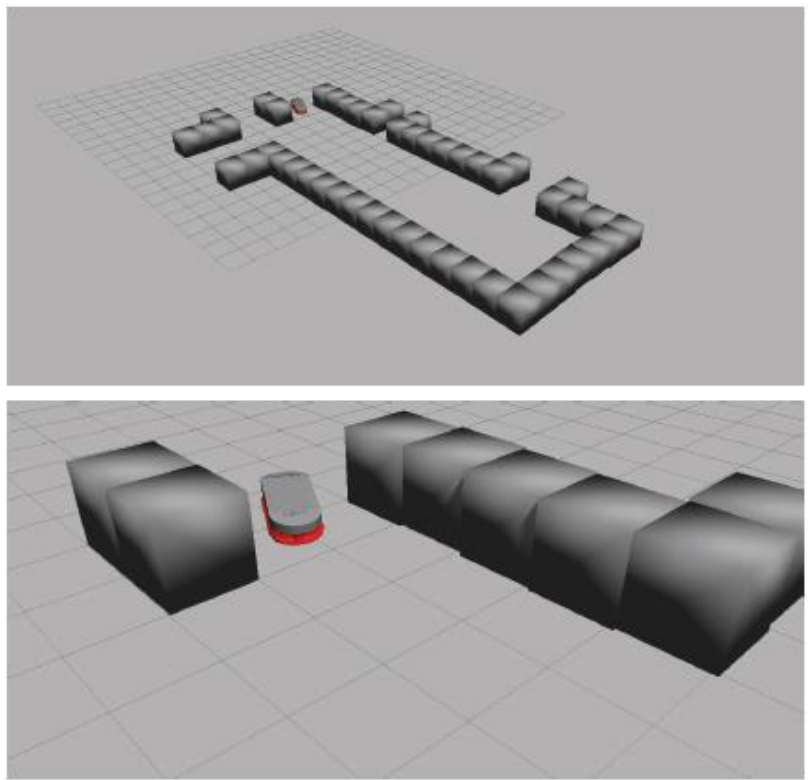

Figura 12. AGV en un ambiente simulado vía Gazebo (Cavanini 2018) [54]

Continuando con GAZEBO como herramienta de simulación de modelos robóticos, (Walenta 2017) [22] propone una solución para la auto-localización, control del comportamiento, enrutamiento libre de conflictos y control de movimiento de una red de AGVs basado en ROS (Walenta 2017) [22]. Esta solución muestra una arquitectura de sistemas para un control descentralizado de múltiples vehículos guiados automatizados que realizan tareas de transporte de materiales en aplicaciones intralogísticas basada en una solución de robótica móvil. Adicionalmente este autor presenta a los vehículos autónomos capaces de ubicarse en un entorno conocido encontrando el camino a través de un gráfico topológico bidireccional discreto, permitiendo dar solución a implicaciones de problemas de planificación global, limita el rango operativo del vehículo, acelera el tiempo de implementación, reduce la complejidad del gráfico topológico y permite una adaptación dinámica a los cambios ambientales, realizando una gestión de tráfico predictiva basada en la comunicación entre los vehículos tratando de maximizar el flujo del material. Un resultado interesante que se puede destacar a partir de lo mostrado en la simulación con GAZEBO es que sugiere mejorar la repetitividad de la posición debido a que en algunos casos presenta una desviación alta con la posición programada. Sin embargo, esto se puede mitigar adicionando más sensores que ayuden al vehículo a ser más preciso en dicha variable.

Precisamente, una posible solución al tema de la posición de un robot en un recinto cerrado usando ROS es la presentada por (Abhishek 2017) [56], el cual diseña un vehículo autónomo que es capaz de mantener una velocidad constante y un monitoreo o vigilancia de la distancia recorrida (Abhishek 2017) [56]. Este autor implementa ROS para realizar rastreo y telemetría de la trayectoria, implementando técnicas de procesamiento de imágenes para la navegación y detección de obstáculos. Adicionalmente, implementa redes neuronales para detectar el camino más corto usando la adquisición de imágenes y diferentes controladores PI y PID para el movimiento y la evasión de obstáculos. Todas estas técnicas de control clásico, control inteligente, procesamiento de imágenes, entre otras herramientas utilizadas por ROS ayudan a que el robot perciba el entorno por donde está transitando, el cual de entrada ya es una tarea compleja debido a la gran cantidad de datos que se recolectan, donde muchos pueden ser relevantes o no serlo. Por otro lado, ROS también ayuda al AGV a auto-localizarse en un espacio determinado en donde debe estar preparado para dar un diagnóstico de su estado, incluso si uno de sus sensores se encuentra averiado. 
De acuerdo a lo anterior, es importante destacar que construir un robot para que realice todas las tareas programadas de manera automática, es una tarea significativa. Sin embargo, cuando un investigador se concentra tanto en la construcción del robot, como en la del software, el tiempo invertido en ambas actividades se puede convertir, tanto para el desarrollo e implementación del AGV, en una labor muy dispendiosa. Debido a esto, autores como (Rhoades 2017) [57] proponen que el investigador se debe concentrar más en el diseño y desarrollo de software que en la misma plataforma robótica. $Y$ esto se facilita ya que la robótica viene experimentando un cambio de paradigma a medida que los robots han cambiado de estar fijos en el piso de fábrica a plataformas basadas en dispositivos móviles (Rhoades 2017) [57]. El objetivo de estas nuevas bases y estructuras de robots es garantizar que la progresión en la investigación no se vea obstaculizada por la carga de abstracciones inferiores, como la configuración sensorial o los mecanismos de accionamiento y la mecánica, sino que le permita al investigador centrarse en la complejidad de la ejecución de algoritmos de niveles superiores ${ }^{52}$, Rhoades (2017) [57]. $Y$ es aquí donde ROS juega un papel muy importante, ya que se convierte en el "middleware" o puente entre hardware y los algoritmos de alto nivel (software). Un ejemplo de lo anterior es el robot móvil Turtlebot [58], el cual ha impulsado el desarrollo de otras plataformas robóticas especializadas que contienen varios motores, sensores y disposiciones de bastidores de dirección. En general, la diversidad de estas plataformas permite la prueba de varios vehículos autónomos, técnicas de detección y despliegue rápido en el proceso [59].

Un ejemplo de implementación de un proyecto que usa un robot móvil comercial (ya construido) en donde se hace énfasis en el desarrollo del software es el mencionado por (Schueftan 2015) [60]. Este autor presenta una arquitectura de localización y mapeo simultaneo (SLAM) aplicada a la navegación autónoma de un AGV, en este caso en particular un robot KUKA youBot, dentro de un sistema de manufactura flexible (FMS). En la figura 13 se puede observar el esquema propuesto.

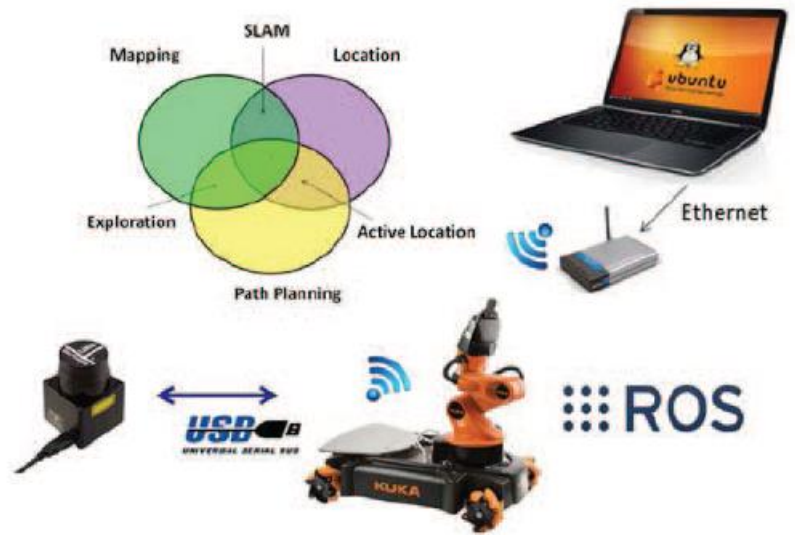

Figura 13. Esquema de arquitectura de control propuesta por (Schueftan 2015) [60]

(Schueftan 2015) [60] expone que uno de los factores claves para integrar una AGV en un FMS está relacionado con las tareas de selección y colocación, donde el AGV es capaz de recoger y entregar objetos en un proceso industrial o logístico determinado. Si se quiere realizar dicha tarea con el vehículo, primero es necesario que reconozca el entorno y luego navegar a través de él autónomamente. De acuerdo a lo anterior, dicho autor utiliza SLAM para dar solución a este problema. Muchos de los algoritmos SLAM citados en la literatura se basan en técnicas probabilísticas utilizando el teorema de Bayes, entre los más usados se puede encontrar el filtro de Kalman (EKF) y por otra parte el filtro de partículas o la estimación de Monte Carlo (Jaulin 2011) [61]. Particularmente este último es una variante de la localización de Markov que se apoya en la representación basada en muestras y resuelve dichos problemas en la localizacion global, en donde esta surge cuando el robot no conoce la posición de inicio y necesita conocer la posición real desde el principio (Aini 2017) [53]

ROS incluye el algoritmo de localización de Monte Carlo, el cual muestra en primera instancia una posición inicial del robot, y luego hace el mapeo para elaborar y precargar el mapa en el robot, el cual es necesario para su localización.

Después de este procedimiento, el robot publica el mapa y este se subscribe a Augmented Monte Carlo Localization (AMCL). Si se completa el mapeo, el robot mide los datos del escaneo laser y los datos de odometría y luego los compara en un editor de mapas. Finalmente, los datos del proceso 
AMCL y el resultado de la comparación son datos de una matriz.

En resumen, hay tres pasos principales para realizar SLAM: percepción, mapeo y navegación. Es en este punto donde ROS toma gran relevancia ya que, usando este software se saca provecho de la sensórica ofrecida por el KUKA youBot, en donde se aplicaron técnicas de fusión de sensores, combinando la información proporcionada por el láser con la odometría del AGV para obtener el espacio escaneado del entorno (Schueftan 2015) [60].

\section{ANÁLISIS Y DISCUSIÓN}

De acuerdo al material revisado se pudo evidenciar la dificultad a la que se enfrentan las organizaciones en el sector comercial, industrial, hospitalario, educativo, entre otros, para la manipulación y transporte de los materiales desde un lugar a otro o incluso de una máquina a otra, teniendo en cuenta que la materia prima puede presentar tamaños y formas irregulares, además de los espacios reducidos o de difícil acceso en donde un usuario debe transitar para transportar los productos a diferentes lugares del sitio de trabajo (Drira 2007) [24].

En la industria frecuentemente surge la necesidad de automatizar o proporcionar una solución para el manejo y transporte de materiales. Dependiendo del tipo de material que se manipule, existen diferentes soluciones en el mercado. Por ejemplo, se pueden encontrar transportadores de banda o rodillos, elevadores, robots para la manipulación de materiales y vehículos guiados autónomos (Martinez 2010) [62]. Este es el caso del transporte de material de forma horizontal, donde los transportadores de bandas o rodillos motorizados son una posible solución. Sin embargo, presentan la desventaja de ser fijos en el lugar instalado y difícilmente se puede variar el diseño en el tiempo, porque desde su esquema inicial se concibe para un lugar y un trabajo específico. Para sitios donde los espacios son reducidos y es necesario aprovechar cada centímetro del lugar de operación para ubicación de maquinaria y puestos de trabajo, o se tiene el riesgo de que los usuarios corran con algún daño físico, se hace necesario el uso de vehículos guiados autónomos, los cuales se ajustan fácilmente para realizar dichas tareas (Ahmed 2015) [43].
Este tipo de tecnología se ha convertido en pieza fundamental en algunas industrias o procesos, tales como centros logísticos en donde se manipulan productos de grandes tamaños y cantidades (Sabattini 2017) [37], y en sistema de transportes urbanos (Osman 2017) [32]. En cada uno, respectivamente, se usan diferentes técnicas para implementar estos vehículos de acuerdo al entorno donde se vayan a desempeñar.

Particularmente en la industria, la manipulación y el transporte de la materia prima de un lugar a otro se ha convertido en factores críticos dentro del flujo productivo, impactando notablemente en el crecimiento industrial y consecuentemente la adquisición de nuevas tecnologías, para darle respuesta a dicha demanda (Samaranayake 2017) [1], (Paul 2017) [63], con lo cual se ha despertado el interés investigativo desde diversas aristas que proponen diferentes alternativas de aplicación de AGV's en la industria (Martinez 2010) [62].

De ahí que el enfoque de este estudio se base en Ios AGV como una solución que ofrece una forma de llevar materia prima de un sitio a otro, ya que los sistemas de manufactura flexible (FMS) son organizados en estaciones de trabajo logrando que los AGV sean clave esencial en este tipo de metodología, en adición a la flexibilidad y adaptabilidad que manejan gracias a la poca infraestructura que necesitan (Ribas 2014) [64], lo cual es muy conveniente debido a que muchas industrias presentan problemas en su disposición interna bien sea por localidad, falta de diseño al inicio de su conformación, distribución desordenada de maquinaria, entre otros (Gutta 2018) [25].

Por otro lado, esto llevará a que los vehículos de guiado autónomo enfrenten situaciones tales como posiciones indeterminadas en el proceso, condiciones desfavorables de tráfico, embotellamiento, rutas no disponibles, entre otros (Paul 2006) [65]. Sin embargo, autores como (Vavrík 2017) [66] y como los otros ya mencionados, proponen una solución de navegación que le permitirá al vehículo conocer a priori el lugar por donde está transitando y (Riesa 2017) [12] plantea algunos métodos de control que ayudarán al móvil a mantener una velocidad y control de la dirección de acuerdo al sitio que se encuentre recorriendo. De esta manera se tendrá un punto de partida para implementar un AGV con ciertas características específicas para el transporte de mercancía junto con el grupo operativo, 
minimizando tiempos improductivos y asegurando la integridad del producto transportado.

Finalmente es importante complementar, en el contexto de la industria 4.0 como los vehículos de guiado autónomos desafían las tecnologías actuales de comunicación y localización. Debido a los requisitos de latencia y confiabilidad, algunas de las soluciones existentes no son capaces de cumplir con el rendimiento requerido por las aplicaciones de automatización industrial. Es por esto que se hace necesario tecnologías de comunicación más flexibles que puedan coexistir en armonía con las diferentes aplicaciones robóticas y contribuyan con un mejor desempeño de los AGV en aplicaciones industriales, y no por el contrario, incapaciten $y / 0$ generen conflicto con otros sistemas de información. Hay que resaltar también que existen otro tipo de vehículos no tripulados para el transporte de materiales y/o mercancía de forma aérea, como lo son los drones, los cuales en aplicaciones de logística tienen requisitos completamente diferentes en comparación con los AGV (Fellan 2018) [67]. Por tal motivo, no son tratados en este documento.

\section{CONCLUSIONES}

Este trabajo presenta una revisión de literatura que se concentró en los AGV desde cuatro puntos de vista (1) identificación y reconocimiento del lugar de trabajo, (2) desempeño de funcionamiento en diferentes entornos, como por ejemplo en sitios cerrados y aire libre, (3) control del AGV y finalmente (4) una breve descripción del papel que juegan los sistemas de software, haciendo énfasis en ROS, para el desarrollo de los algoritmos que permiten el funcionamiento del vehículo.

Se presentan los AGV de acuerdo a la solución que ofrecen para el trasporte de materia prima, mercancía, objetos, entre otros, de acuerdo a la necesidad de automatizar procesos, mejorar tiempos, flujos de producción y aseguramiento de la integridad del producto y del recurso humano.

Se reconoce claramente las oportunidades que ofrece esta tecnología en el área de la robótica y por eso es necesario seguir profundizando e investigando en herramientas de software como ROS, que ayuden a potenciar los diferentes recursos de hardware que componen a un robot logrando así maximizar su funcionamiento y prestación de un mejor servicio en el sitio de aplicación. También se evidencia la influencia de los AGV dentro del contexto de la Industria 4.0, y como juegan un papel importante en el mejoramiento de los flujos productivos gracias al intercambio de información con los sistemas de gestión y quienes los administran para la toma de decisiones.

De igual manera cabe resaltar que se han logrado avances significativos en el transporte de materiales, sin embargo, es necesario seguir investigando debido a que diariamente surgen nuevas ideas para minimizar tiempos de transporte de productos y flujos de proceso, por lo que se hace necesario seguir creciendo a nivel tecnológico de manera que se pueda satisfacer al usuario final.

\section{AGRADECIMIENTOS}

Se agradece en primera instancia a C.I. G \& Lingerie S.A.S por brindar el espacio y las herramientas para el desarrollo de este proyecto en sus instalaciones y a los profesores que han asesorado del Politécnico Jaime Isaza Cadavid.

\section{REFERENCIAS BIBLIOGRÁFICAS}

1. Samaranayake $P$, Ramanathan $K$, Laosirihongthong $\mathrm{T}$. Implementing industry $4.0-A$ technological readiness perspective. 2017 IEEE Int Conf Ind Eng Eng Manag. 2017:529-533. doi:10.1109/IEEM.2017.8289947

2. Hill R, Devitt J, Anjum A, Ali M. Towards InTransit Analytics for Industry 4 . 0. In: 2017 IEEE International Conference on Internet of Things (IThings) and IEEE Green Computing and Communications (GreenCom) and IEEE Cyber, Physical and Social Computing (CPSCom) and IEEE Smart Data (SmartData). ; 2017:810-817. doi:10.1109/iThings-GreenCom-CPSComSmartData.2017.124

3. Vasiljević G, Miklić D, Draganjac I, Kovačić $Z$, Lista P. High-accuracy vehicle localization for autonomous warehousing. Robot Comput Integr Manuf. 2016;42:1-16. doi:10.1016/j.rcim.2016.05.001

4. Chen B, Qu T, Thurer M, Huang GQ, Li C, $\mathrm{Xu}$ S. Warehouse workload control for 
production logistic. In: 2017 13th IEEE Conference on Automation Science and Engineering (CASE). ; 2017:237-242. doi:10.1109/COASE.2017.8256109

5. Wang HF, Chang CM. Facility layout for an automated guided vehicle system. Procedia Comput Sci. 2015;55(Itqm):52-61. doi:10.1016/j.procs.2015.07.007

6. Bechtsis D, Tsolakis N, Vlachos D, lakovou E. Sustainable supply chain management in the digitalisation era: The impact of Automated Guided Vehicles. J Clean Prod. 2017; 142:3970-3984.

doi:10.1016/j.jclepro.2016.10.057

7. Bostelman R, Messina E. Towards Development of an Automated Guided Vehicle Intelligence Level Performance Standard. In: Autonomous Industrial Vehicles: From the Laboratory to the Factory Floor. 2016:1-22. doi:10.1520/STP159420150054

8. Le-Anh T, De Koster MBM. A review of design and control of automated guided vehicle systems. Eur $J$ Oper Res. 2006;171(1):1-23.

doi:10.1016/j.ejor.2005.01.036

9. Andreasson $\mathrm{H}$, Bouguerra $\mathrm{A}$, Cirillo $\mathrm{M}$, et al. Autonomous transport vehicles: Where we are and what is missing. IEEE Robot Autom Mag. 2015;22(1):34-75. doi:10.1109/MRA.2014.2381357

10. Shakya R, Rajanwal K, Patel S, Maurya RK. Optimization and Designing of Pid, Fuzzy \& Pid-Fuzzy Controller. Int J Sci Eng Res. 2014;5(1):2040-2048.

11. Zulfatman, Rahmat MF. Application of SelfTuning Fuzzy Pid Controller on Industrial Hydraulic Actuator. Nternational J Smart Sens Intell Syst. 2009;2(2):246-261.

12. S RKA. Speed Control System Design Using Fuzzy-PID For Load Variation of Automated Guided Vehicle ( AGV ). In: 2017 2nd International Conference on Frontiers of Sensors Technologies. ; 2017:5-9. doi:10.1109/ICFST.2017.8210549

13. Juan Martin Echeverri Estrada PAEM. Caracterización De Un Agv (Vehículo Guiado Automáticamente) En El Sistema De Manufactura Flexible; Caso Centro Tecnológico De Automatización Ctai De La Pontificia Universidad Javeriana. 2012. http://repository.javeriana.edu.co/bitstream/1 0554/10296/1/EcheverriEstradaJuanMartin2 013.pdf.
14. Vis IFA. Survey of research in the design and control of automated guided vehicle systems. Eur J Oper Res. 2006;170(3):677709. doi:10.1016/j.ejor.2004.09.020

15. Gunter Ullrich. Automated Guided Vehicle Systems. Springer. Germany; 2015. doi:10.1016/0166-3615(84)90043-5

16. Theunissen J. Smart AGV System for Manufacturing Shopfloor in the Context of Industry 4 . 0. In: 2018 25th International Conference on Mechatronics and Machine Vision in Practice (M2VIP). IEEE; 2018:1-6. doi:10.1109/M2VIP.2018.8600887

17. Chen B. VERIFICATION AND VALIDATION STRATEGIES ON COLLABORATIVE ROBOTIC. In: 2017 IEEE International Symposium on Product Safety and Compliance Engineering - Taiwan (ISPCETW). IEEE; 2017:1-2. doi:10.1109/ISPCETW.2017.8626833

18. Parys $R$ Van, Verbandt $M$, Kotz $M$, et al. Distributed Coordination, Transportation \& Localisation in Industry 4 . 0. In: 2018 International Conference on Indoor Positioning and Indoor Navigation (IPIN). ; 2018:24-27. doi:10.1109/IPIN.20188533768

19. Julián $A$, Zapata M, Felipe D, Lema G, Castro Ospina AE. Diseño De Prototipo De Un Vehículo De Guiado Automático.; 2015.

20. Bacik J, Durovsky F, Biros M, Kyslan K, Perdukova D, Sanjeevikumar P. Pathfinder Development of Automated Guided Vehicle for Hospital Logistics. IEEE Access. 2017;5:1-1.

doi:10.1109/ACCESS.2017.2767899

21. Kumar Das S. Design and Methodology of Automated Guided Vehicle-A Review. IOSR $J$ Mech Civ Eng. 2016;03(03):29-35. doi:10.9790/1684-15010030329-35

22. Walenta $R$, Schellekens $T$, Ferrein $A$, Schiffer S. A decentralised system approach for controlling AGVs with ROS. In: 2017 IEEE AFRICON: Science, Technology and Innovation for Africa, AFRICON 2017. ; 2017:1436-1441.

doi:10.1109/AFRCON.2017.8095693

23. Garber L. Robot OS : A New Day for Robot Design. IEEE Journals Mag. 2013;46(12):1620. doi:10.1109/MC.2013.434

24. Drira A, Pierreval H, Hajri-Gabouj S. Facility layout problems: A survey. Annu Rev Control. 2007;31(2):255-267. doi:10.1016/j.arcontrol.2007.04.001

25. Reddy Gutta P, Sai Chinthala V, Venkatesh 
Manchoju R, Charan MVN V, Purohit R. A Review On Facility Layout Design Of An Automated Guided Vehicle In Flexible Manufacturing System. Mater Today Proc. 2018;5(2):3981-3986.

doi:10.1016/j.matpr.2017.11.656

26. Beinschob P, Meyer M, Reinke C, Digani V, Secchi C, Sabattini L. Semi-automated map creation for fast deployment of AGV fleets in modern logistics. Rob Auton Syst. 2017;87:281-295.

doi:10.1016/j.robot.2016.10.018

27. Lu S, Xu C, Zhong RY, Wang L. A RFIDenabled positioning system in automated guided vehicle for smart factories. J Manuf Syst. 2017;44:179-190. doi:10.1016/j.jmsy.2017.03.009

28. Saab SS, Nakad ZS. A Standalone RFID Indoor Positioning System Using Passive Tags. IEEE Trans Ind Electron. 2011;58(5):1961-1970.

doi:10.1109/TIE.2010.2055774

29. Lu S, Xu C, Zhong RY, Wang L. A passive RFID tag-based locating and navigating approach for automated guided vehicle. Comput Ind Eng. 2018;(xxxx):0-1. doi:10.1016/j.cie.2017.12.026

30. Hartmann S. Scheduling of Automated Guided Vehicle in Different Flexible Manufacturing System Environment. Int $\mathrm{J}$ Innov Res Adv Eng. 2014;1(8):262-267.

31. Kang J, Lee J, Eum $\mathrm{H}$, Hyun $\mathrm{CH}$, Parks $\mathrm{M}$. An application of parameter extraction for AGV navigation based on computer vision. In: 2013 10th International Conference on Ubiquitous Robots and Ambient Intelligence, URAI 2013. ; 2013:622-626. doi:10.1109/URAI.2013.6677408

32. Osman K, Ghommam J, Saad M. Combined road following control and automatic lane keeping for automated guided vehicles. 2016 14th Int Conf Control Autom Robot Vision, ICARCV 2016. 2017;2016(November):13-15. doi:10.1109/ICARCV.2016.7838680

33. Pedan $M$, Gregor M, Plinta D. Implementation of Automated Guided Vehicle System in Healthcare Facility. Procedia Eng. 2017;192:665-670. doi:10.1016/j.proeng.2017.06.115

34. Acosta Calderon CA, Mohan ER, Ng BS. Development of a hospital mobile platform for logistics tasks. Digit Commun Networks. 2015;1(2):102-111. doi:10.1016/j.dcan.2015.03.001

35. Barnea A, Berrabah SA. IMU ( Inertial Measurement Unit ) Integration for the Navigation and Positioning of Autonomous Robot Systems. CEAI. 2011;13(2):38-43.

36. Evany Ricardo Sepúlveda Gómez. Diseño e implementación de un vehículo guiado autónomo para la ubicación de libros en un ambiente controlado. 2014. www.springer.com.

37. Lorenzo Sabattini, Mika Aikio, Patric Beinschob, Elena Cardarelli VD. The PANRobots Project: Advanced Automated Guided Vehicle Systems for Industrial Logistics. IEEE Robot Autom Mag. 2017;18(March 2018):55-64. doi:10.1109/MRA.2017.2700325

38. Wurman PR, D'Andrea R, Mountz $M$. Coordinating Hundreds of Cooperative, Autonomous Vehicles in Warehouses. Al Mag. 2008;29(1):9. doi:10.1609/aimag.v29i1.2082

39. Valencia-Hernández C. A., RestrepoMartínez A., Muñoz-Ceballos N. D., "Caracterización de marcadores de realidad aumentada para suuso en robótica," Revista Politécnica, vol. 13, no. 25, pp. 87-102, 2017. doi:10.33571/rpolitec.v13n25a7

40. Parikh P, Sheth S, Vasani R, Gohil JK. Implementing Fuzzy Logic Controller and PID Controller to a DC Encoder Motor - "a case of an Automated Guided Vehicle." Procedia Manuf. 2018; 20:219-226. doi:10.1016/j.promfg.2018.02.032

41. Rusdinar A, Kim S-S. Modeling of vision based robot formation control using fuzzy logic controller and extended Kalman filter. Int J Fuzzy Log Intell Syst. 2012;12(3):238244. doi:10.5391/IJFIS.2012.12.3.238

42. Kudinov YI, Kolesnikov VA, Pashchenko FF, Pashchenko AF, Papic L. Optimization of Fuzzy PID Controller's Parameters. Procedia Comput Sci. 2017;103(October 2016):618622. doi:10.1016/j.procs.2017.01.086

43. Ahmed SA, Petrov MG. Trajectory Control of Mobile Robots using Type-2 Fuzzy-Neural PID Controller. IFAC-PapersOnLine. 2015;48(24):138-143. doi:10.1016/j.ifacol.2015.12.071

44. M. B. Nugraha, Rizki Ardianto P. DD. Design and Implementation of RFID Line-Follower Robot System with Color Detection Capability using Fuzzy Logic. In: 2015 
International Conference on Control, Electronics, Renewable Energy and Communications (ICCEREC). ; 2015:75-78. doi:10.1109/ICCEREC.2015.7337058

45. Zou O, Member RYZ. Automatic Logistics in a Smart Factory using RFID-enabled AGVs. In: 2018 IEEE/ASME International Conference on Advanced Intelligent Mechatronics (AIM). IEEE; 2018:822-826. doi:10.1109/AIM.2018.8452349

46. Bui TL, Doan PT, Kim HK, Kim SB. Trajectory tracking controller design for AGV using laser sensor based positioning system. In: 2013 9th Asian Control Conference (ASCC). 2013:1-5. doi:10.1109/ASCC.2013.6606202

47. Carlucho I, De Paula M, Villar SA, Acosta GG. Incremental Q-learning strategy for adaptive PID control of mobile robots. Expert Syst Appl. 2017;80:183-199. doi:10.1016/j.eswa.2017.03.002

48. Hellmund A-M, Wirges S, Tas ÖS, Bandera C, Salscheider NO. Robot Operating System: A Modular Software Framework for Automated Driving. In: Proc. IEEE International Conference on Intelligent Transportation Systems (ITSC). ; 2016:1564-1570.

doi:10.1109/ITSC.2016.7795766

49. Messner J. EB Assist ADTF Automotive Data and Time Triggered Framework.; 2015.

50. K. Hoffmeister. Automated Driving Necessary Infrastructure Shift. ATZ Electron. 2016;1:42-47.

doi:https://doi.org/10.1007/s38314-0160012-z

51. Quigley M, Conley K, Gerkey B, et al. ROS: an open-source Robot Operating System. Icra. 2009;3(Figure 1):5. http://pub1.willowgarage.com/ konolige/cs2 25B/docs/quigley-icra2009-ros.pdf.

52. Robot Operating System (ROS). http://www.ros.org/. Accessed October 8, 2018.

53. Aini FRQ, Jati AN, Sunarya U. A study of Monte Carlo localization on robot operating system. In: 2016 International Conference on Information Technology Systems and Innovation, ICITSI 2016 - Proceedings. ; 2017:1-6. doi:10.1109/ICITSI.2016.7858235

54. Cavanini L, Cicconi P, Freddi A, et al. A Preliminary Study of a Cyber Physical System for Industry 4.0: Modelling and CoSimulation of an AGV for Smart Factories.
In: 2018 Workshop on Metrology for Industry 4.0 and IOT. IEEE; 2018:169-174. doi:10.1109/METROI4.2018.8428334

55. COOJA Simulator. http://www.contikios.org/start.html. Accessed October 10, 2018.

56. Abhishek B, Varun Rufus Raj Samuel D, U.V. Vignesh, Gautham S, Keshav k SRN. ROS based Stereo Vision System for Autonomous Vehicle. In: IEEE International Conference on Power, Control, Signals and Instrumentation Engineering (ICPCSI-2017). ; 2017:2269-2273. doi:10.1109/ICPCSI.2017.8392121

57. Rhoades BB, Sabo JP, Conrad JM. Enabling a National Instruments DaNI 2.0 robotic development platform for the Robot Operating System. In: Conference Proceedings - IEEE SOUTHEASTCON. ; 2017:1-5.

doi:10.1109/SECON.2017.7925293

58. TurtleBot.

http://emanual.robotis.com/docs/en/platform/ turtlebot3/overview/. Accessed October 15, 2018.

59. Robotnik-Portafolio. https://www.robotnik.es/logistics/portfolio/. Accessed October 15, 2018.

60. Schueftan DS, Colorado MJ, Bernal IFM. Indoor mapping using SLAM for applications in Flexible Manufacturing Systems. In: 2015 IEEE 2nd Colombian Conference on Automatic Control, CCAC 2015 - Conference Proceedings. doi:10.1109/CCAC.2015.7345226

61. Jaulin L. Range-only SLAM with occupancy maps: A set-membership approach. IEEE Trans Robot. 2011;27(5):1004-1010. doi:10.1109/TRO.2011.2147110

62. Martinez-Barbera $H$, Herrero-Perez $D$. Development of a flexible AGV for flexible manufacturing systems. Ind Robot An Int $\mathrm{J}$. 2010;37(5):459-468.

doi:10.1108/01439911011063281

63. Paul PV, Saraswathi R. The Internet of Things - A Comprehensive Survey. In: 2017 International Conference on Computation of Power, Energy, Information and Communication (ICCPEIC). ; 2017:421-426.

64. Ribas-Xirgo L. A Virtual Laboratory of a Manufacturing Plant operated with mobile robots. In: Proceedings of the 2014 IEEE Emerging Technology and Factory Automation (ETFA). ; 2014:1-4. 
doi:10.1109/ETFA.2014.7005279

65. Paul G, Liu DK. Replanning of Multiple Autonomous Vehicles in Material Handling. Proc IEEE Int Conf Robot Autom Mechatronics, RAM. 2006:231-236. doi:10.1109/RAMECH.2006.252729

66. Vavrík V, Gregor M, Grznár P. Computer Simulation as a Tool for the Optimization of Logistics Using Automated Guided Vehicles. Procedia Eng. 2017;192:923-928. doi:10.1016/j.proeng.2017.06.159

67. Fellan A, Schellenberger C, Zimmermann M, Schotten HD. Enabling Communication Technologies for Automated Unmanned Vehicles in Industry 4 . 0. In: 2018 International Conference on Information and Communication Technology Convergence (ICTC). IEEE; 2018:171-176. doi:10.1109/ICTC.2018.8539695 